\title{
PECULIAR PROPERTIES OF ULTRATHIN FCC IRON FILMS EPITAXIALLY GROWN ON $\mathrm{Cu}(001)$
}

\author{
R. WOJNECKI ${ }^{a *}$ AND A. SUKIENNICKI ${ }^{a, b}$ \\ ${ }^{a}$ Institute of Physics, Polish Academy of Sciences \\ Al. Lotników 32/46, 02-668 Warsaw, Poland \\ ${ }^{b}$ Faculty of Physics, Warsaw University of Technology \\ Koszykowa 75, 00-662 Warsaw, Poland
}

(Received November 3, 1999)

\begin{abstract}
Very peculiar electronic structure and local magnetic moment distribution for fcc Fe ultrathin films of 1, 2, 3, and 4 monolayers of thickness, epitaxially grown on $\mathrm{Cu}(001)$ substrates and covered with some layers of $\mathrm{Cu}$, are calculated. The lattice constant of the Fe film is assumed to be equal to that of $\mathrm{Cu}$, homogeneously in the whole film. The competition between the surface ferromagnetism and bulk antiferromagnetism results in ferromagnetism of 2-monolayer film, asymmetric solution for 3-monolayer film and antisymmetric solution for 4-monolayer film.
\end{abstract}

PACS numbers: $75.70 . \mathrm{Ak}$

\section{Introduction}

Electronic structure and the magnetic properties of ultrathin fcc Fe films epitaxially grown on $\mathrm{Cu}(100)$ substrates are a subject of interest in the last years [1-9]. It has been established very well theoretically [1-4] that two surface monolayers of the Fe films are coupled ferromagnetically, while the inner layers are ordered layer-by-layer antiferromagnetically. It is interesting to check which tendency: surface ferromagnetism or antiferromagnetism inside the film is stronger in the case of ultrathin Fe films of 2,3, or 4 monolayers of thickness. For two monolayers film the answer has been given in Ref. [1] but for films of 3 and 4 monolayers no results are reported up to now.

In the present work, we calculated the electronic structure and the distribution of the local magnetic moments across the film for the epitaxially grown Fe films included between the $\mathrm{Cu}(001)$ substrate and the $\mathrm{Cu}(001)$ covering layers, with the lattice constant assumed to be equal to that of $\mathrm{Cu}$, homogeneously in the whole system $(3.61 \AA)$. The calculations are performed for fcc Fe films of one, two, three, and four monolayers. Periodic boundary conditions in the direction perpendicular to the film are assumed. Such boundary conditions could suggest

\footnotetext{
*e-mail: wojne@ifpan.edu.pl
} 
that multilayer properties are calculated, however we should stress here that these conditions are used only for simplification of our calculations for a single thin film. Similarly as in Ref. [4], the calculations are made self-consistently using the linear muffin-tin orbital (LMTO) method with the von Barth-Hedin approximation for exchange and correlation effects. The relativistic, spin polarized approximation is used. Spin-orbit coupling is included. All $s, p$, and $d$ electrons are taken into account. The calculations were performed using $273 k$ points in the irreducible part of the Brillouin zone for tetragonal lattice. A sufficiently large number of iterations are carried out to have the total energy converged to an accuracy better than $0.05 \mathrm{mRy}$.

\section{Results}

For one monolayer fcc Fe film (a two-dimensional magnetic system) included between 2 monolayers of $\mathrm{Cu}(001)$ substrate and one $\mathrm{Cu}(001)$ monolayer covering, with periodic boundary conditions in direction perpendicular to the film, the ferromagnetic result is obtained with the magnetic moment equal to 2.46 (in Bohr magneton units). This result is comparable with that of Ref. [1] with exception that in their work they have obtained a slightly bigger value (2.60 Bohr magnetons) of the magnetic moment.

For two monolayers thick fcc Fe films included between $\mathrm{Cu}(001)$ substrate simulated by two monolayers of $\mathrm{Cu}$ - and two covering monolayers of $\mathrm{Cu}$, with periodic boundary conditions in direction perpendicular to the film, the state with the lowest total energy corresponds to the ferromagnetic configuration (denoted by us as $(++)$ configuration) with the magnetic moments of both monolayers equal to 2.50 (in Bohr magneton units). Some insignificant magnetic moments are induced in the $\mathrm{Cu}$ layers as the result of proximity effects. Figure 1 depicts the local density of states for both layers considered. The antiferromagnetic configuration (denoted as $(+-)$ configuration) with the magnetic moments of monolayers equal to 2.16 and -2.16 corresponds to higher total energy (by $2 \mathrm{mRy}$ ). Our result shows that the ferromagnetic tendency coming from the surface effects dominates over the antiferromagnetic tendency of the bulk, of course, due to a lack of the bulk material in films of such a small thickness. This result is in a good agreement with that of Ref. [1], the only differences are that magnetic moments in our case are not so enhanced at the surfaces as in Ref. [1] and the difference in the energy between both configurations $((++)$ and $(+-))$ are much smaller in our case.

Extremely interesting results are obtained for three monolayers thick Fe film included between two monolayers of $\mathrm{Cu}$, playing role of a substrate, and one covering $\mathrm{Cu}$ monolayer, with periodic boundary conditions assumed in the direction perpendicular to the film. One could expect that the surface effects could again dominate over the tendency of the bulk and that the ferromagnetic $(+++)$ configuration will be lower in energy than the $(+-+)$ configuration. However, it is not the case: the antiferromagnetic $(+-+)$ configuration with the local magnetic moments of the Fe monolayers $2.16,-1.39,2.16$ has definitely lower total energy than the ferromagnetic one with moments 2.49, 2.32, 2.49 (in Bohr magneton units). One should notice that the values of local moments for the antiferromagnetic $(+-+)$ configuration are strongly reduced in comparison with the corresponding 


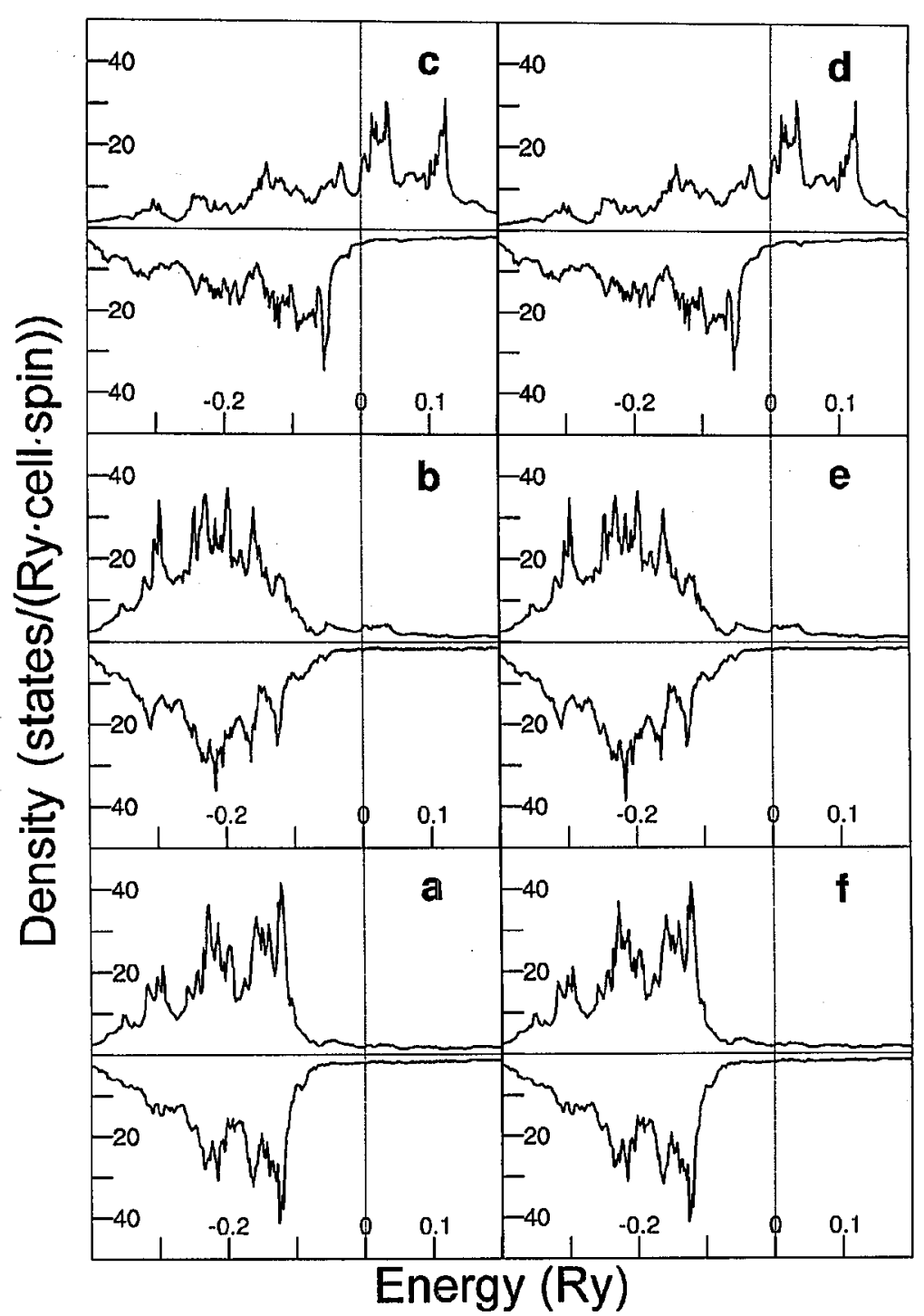

Fig. 1. Density of states for the 2-monolayer Fe film with $(++)$ magnetic configuration: (a) $\mathrm{Cu}$ (next to interface), (b) $\mathrm{Cu}$ (interface), (c) $\mathrm{Fe}$ (interface), (d) $\mathrm{Fe}$ (interface), (e) $\mathrm{Cu}$ (interface), (f) $\mathrm{Cu}$ (next to interface). Scale of energy: from $-0.4 \mathrm{Ry}$ to +0.2 Ry; Fermi level corresponds to zero of energy (shown by vertical line). Scale of DOS: from 0 to 50 states/(Ry-cell-spin) shown by horizontal ticks separated by 10 states/(Ry.cell.spin). Upper parts - spin up, down parts — spin down.

values for the ferromagnetic state. This result shows that the competition between both tendencies mentioned above has more complicated character: due to them some kind of "frustration" occurs. As a result, the asymmetric $(--+)$ configuration has the lower total energy even in comparison with the $(+-+)$ state, 


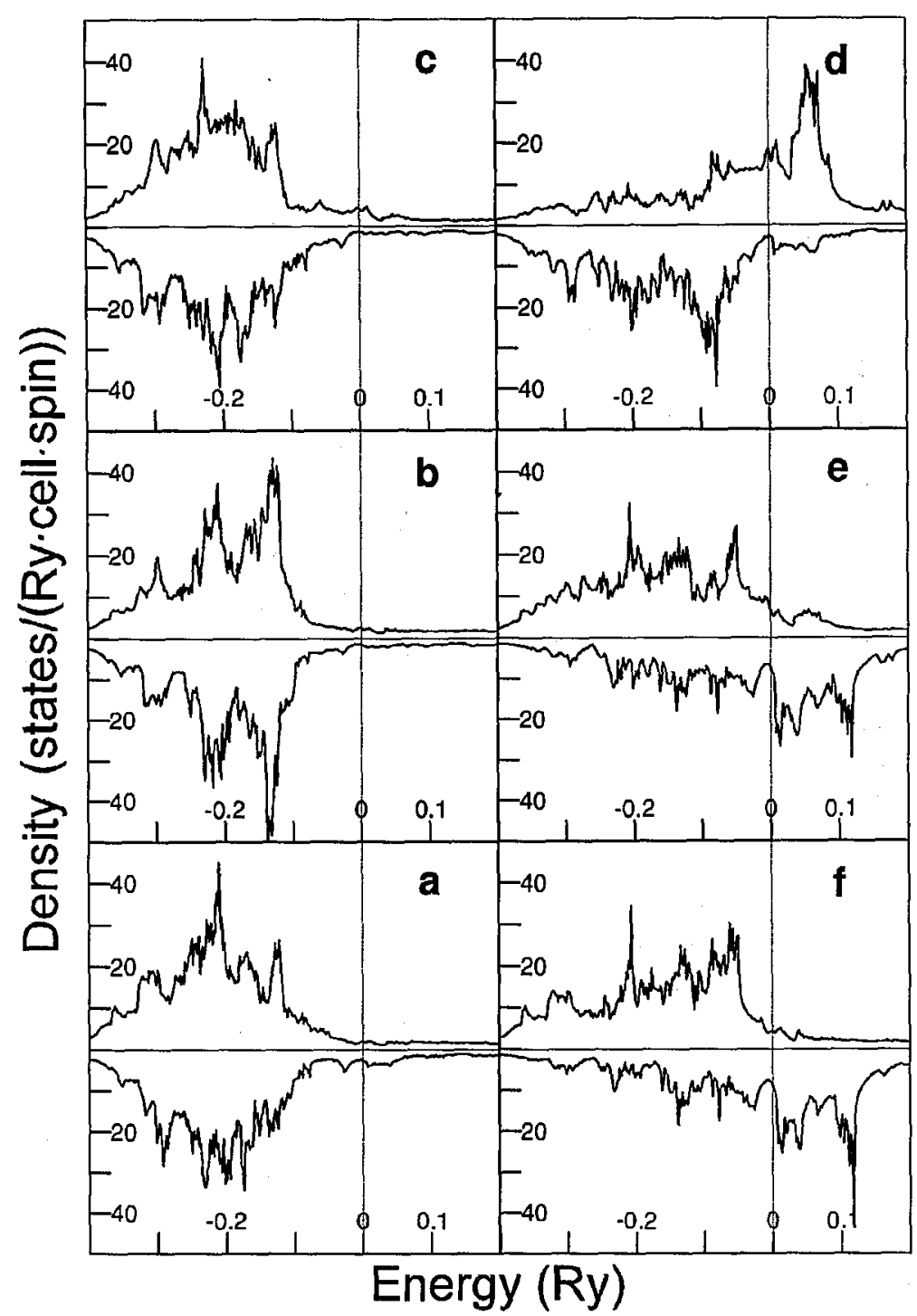

Fig. 2. Density of states for the 3-monolayer Fe film with $(--+)$ magnetic configuration: (a) $\mathrm{Cu}$ (next to interface), (b) $\mathrm{Cu}$ (interface), (c) Fe (interface), (d) Fe (middle), (e) Fe (interface), (f) $\mathrm{Cu}$ (interface). Rest of the caption as for Fig. 1.

although the difference is only $0.7 \mathrm{mRy}$ ! The magnetic moments for the $(--+)$ configuration are also asymmetric: $-2.49,-2.12,2.06$ (in Bohr magneton units) and the local densities of states for $(--+)$ configuration are shown in Fig. 2 and for $(+-+)$ configuration - in Fig. 3. We should like to stress that the differences in energy between $(++t)$ and $(+-+)$ configurations are definitely above inaccuracy of computations, while a difference between energy of $(+-+)$ and $(--+)$ configurations is in the range of error of the LMTO method. 


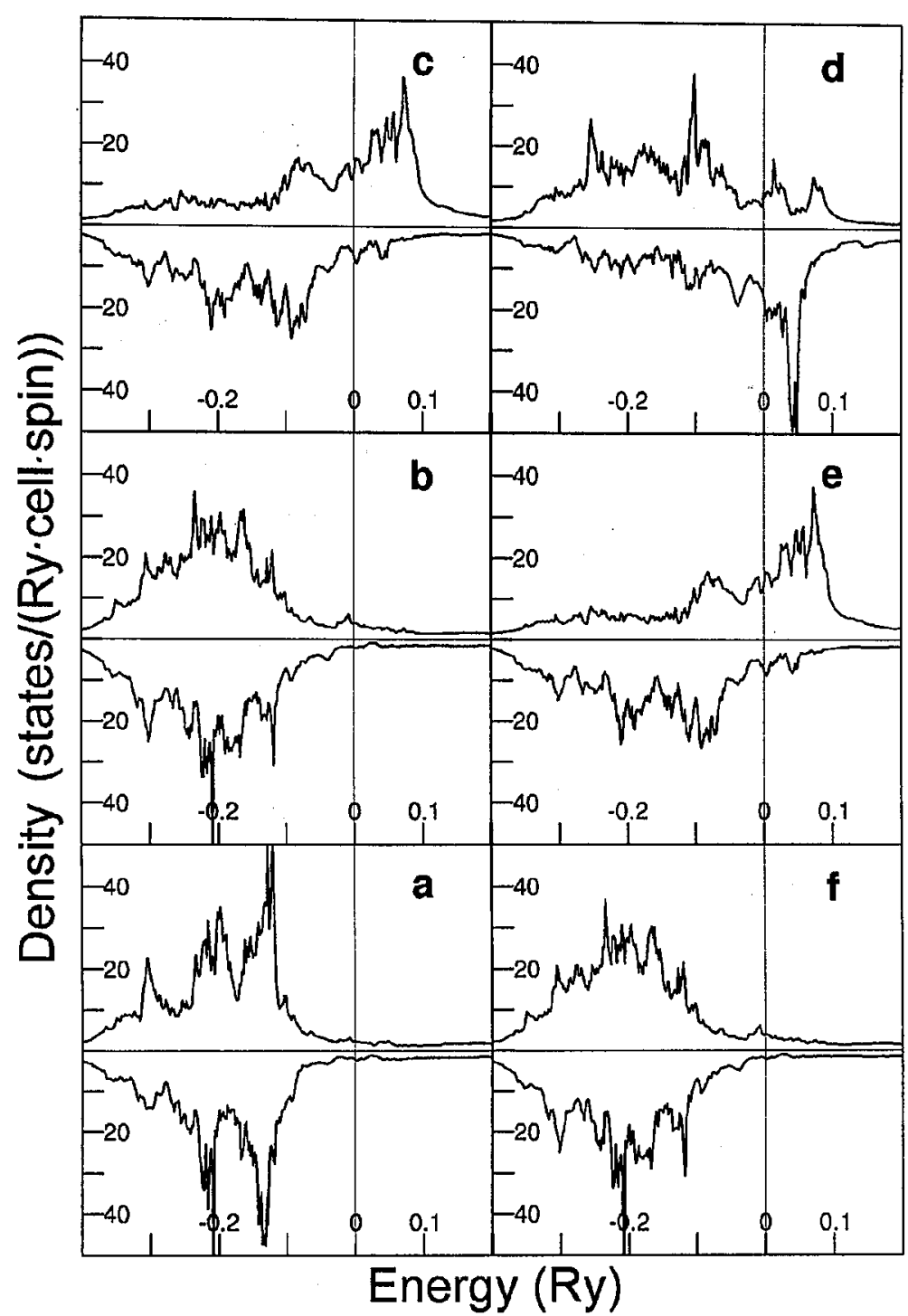

Fig. 3. Density of states for the 3-monolayer Fe film with $(+-+)$ magnetic configuration: (a) $\mathrm{Cu}$ (next to interface), (b) $\mathrm{Cu}$ (interface), (c) Fe (interface), (d) $\mathrm{Fe}$ (middle), (e) $\mathrm{Fe}$ (interface), (f) $\mathrm{Cu}$ (interface). Rest of the caption as for Fig. 1.

The results obtained for the four-monolayer Fe film included between two $\mathrm{Cu}$ monolayers of the substrate and two covering $\mathrm{Cu}$ monolayers (with periodic boundary conditions across the film) are also interesting: both tendencies described above lead in this case to the antisymmetric $(++--)$ configuration being the state with the lowest total energy. Both surface regions are ordered ferromagnetically inside of them but antiferromagnetically each to another. Figure 4 gives the local densities of states, the resulting magnetic moments are 2.47, 2.01, -2.01 


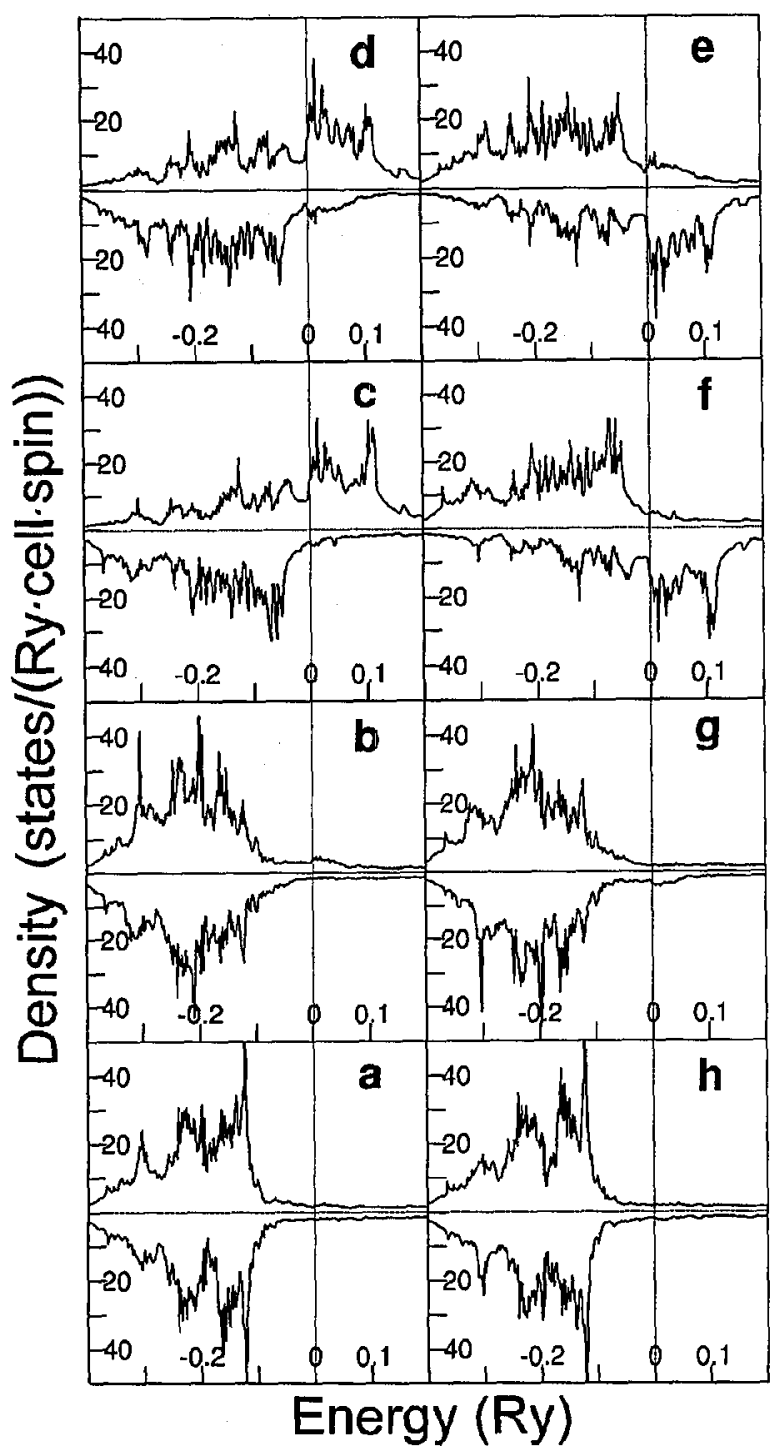

Fig. 4. Density of states for the 4-monolayer Fe film with $(++--)$ magnetic configuration: (a) $\mathrm{Cu}$ (next to interface), (b) $\mathrm{Cu}$ (interface), (c) Fe (interface), (d) Fe (middle), (e) $\mathrm{Fe}$ (middle), (f) $\mathrm{Fe}$ (interface), (g) $\mathrm{Cu}$ (interface), (h) $\mathrm{Cu}$ (next to interface). Rest of the caption as for Fig. 1.

and -2.47 (in Bohr magneton units). We should stress however that a difference in total energy between $(++--)$ and $(+--+)$ configurations is very small $(0.7 \mathrm{mRy})$ which is again in the range of error in our calculations. Moreover, our results are very sensitive with respect to changes of the lattice constant assumed for calculations. Figure 5 depicts the influence of these changes for few types of configurations. Generally speaking, changes of the lattice constant may cause the 


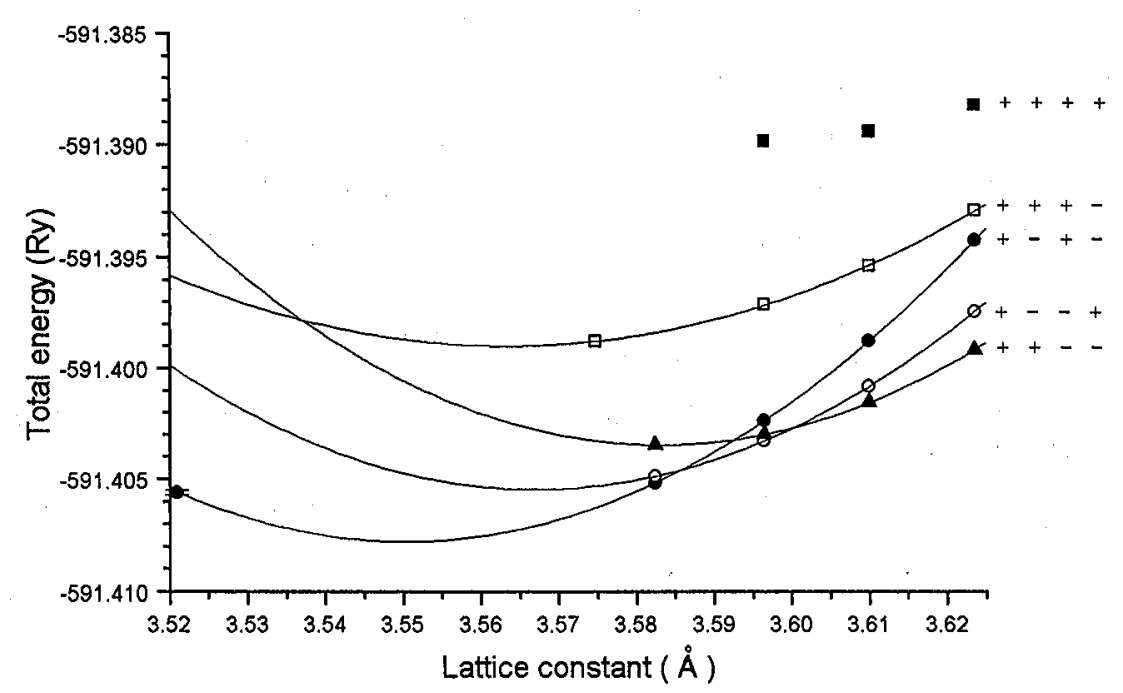

Fig. 5. Total energy dependence from lattice constant for different magnetic configurations. Points show values calculated by LMTO method, parabolas are fitted.

change of the lowest-energy configuration. In particular, the change of the lattice constant from the value characteristic of copper $(3.61 \AA)$ leads to the lowest energy configuration $(+--+)$. This result, however, is in the range of error of the LMTO method. Moreover, our LMTO calculations give for pure copper the lattice constant $3.582 \AA$ instead of the experimental value $3.61 \AA$. This is a very small $(0.8 \%)$ error, however, it causes that the lowest energy configuration for the calculated lattice constant is $(+-+-)$. In this case also the result is in the range of error of the LMTO method. The most important results, however, are those obtained for the experimental lattice constant of copper.

\section{Conclusion}

We conclude that the competition between the ferromagnetic surface effects and the antiferromagnetism of the inner parts of the fcc Fe film leads to the interesting phenomena when the considered film is thin enough. Thickness of the Fe film is a parameter which controls a mutual importance of both effects and for 2-monolayer Fe film the ferromagnetic state is obtained, for 3-monolayer Fe film the asymmetric solution has the lowest energy, and for 4-monolayer Fe film antisymmetric configuration is the best solution.

Important result is that for cases where the both effects have the comparable influences, asymmetric solutions have to be allowed.

The solution found in the case of four Fe monolayers could have a practical importance. Antisymmetric configuration could be easily magnetized by the external field to the ferromagnetic configuration of the whole film, leading to the magnetoresistance like for the standard multilayer films. 


\section{References}

[1] C.L. Fu, A.J. Freeman, Phys. Rev. B 35, 925 (1987).

[2] G.W. Fernando, B.R. Cooper, Phys. Rev. B 38, 3016 (1988).

[3] T. Kraft, P.M. Marcus, M. Scheffler, Phys. Rev. B 49, 11511 (1994).

[4] R. Wojnecki, A. Sukiennicki, Acta Phys. Pol. A 93, 737 (1998).

[5] W.A.A. Macedo, W. Keune, Phys. Rev. Lett. 61, 475 (1988).

[6] J. Thomassen, F. May, B. Feldmann, M. Wuttig, H. Ibach, Phys. Rev. Lett. 69, 3831 (1992).

[7] Dongqi Li, M. Freitag, J. Pearson, Z.Q. Qiu, S.D. Bader, Phys. Rev. Lett. 72, 3112 (1994).

[8] M. Donath, B. Gubanka, F. Passek, V. Dose, Europhysics Conf. Abstr. 20A, 143 (1996).

[9] M. Matsui, M. Doi, A. Kida, Y. Yamada, J. Appl. Phys. 79, 5883 (1996) (abstract only). 